\title{
The influence of spacecraft latitudinal offset on the accuracy of corotation forecasts
}

Article

Published Version

Creative Commons: Attribution 4.0 (CC-BY)

Open Access

Turner, H. ORCID: https://orcid.org/0000-0002-4012-8004, Owens, M. J. ORCID: https://orcid.org/0000-0003-2061-2453, Lang, M. S. ORCID: https://orcid.org/0000-0002-1904-3700 and Gonzi, S. ORCID: https://orcid.org/0000-0002-0974-7392 (2021) The influence of spacecraft latitudinal offset on the accuracy of corotation forecasts. Space Weather, 19 (8). e2021SW002802. ISSN 1542-7390 doi: https://doi.org/10.1029/2021SW002802 Available at https://centaur.reading.ac.uk/99516/

It is advisable to refer to the publisher's version if you intend to cite from the work. See Guidance on citing.

Published version at: http://dx.doi.org/10.1029/2021SW002802

To link to this article DOI: http://dx.doi.org/10.1029/2021SW002802

Publisher: American Geophysical Union

All outputs in CentAUR are protected by Intellectual Property Rights law, including copyright law. Copyright and IPR is retained by the creators or other copyright holders. Terms and conditions for use of this material are defined in the End User Agreement. 


\section{www.reading.ac.uk/centaur}

\section{CentAUR}

Central Archive at the University of Reading

Reading's research outputs online 


\section{Space Weather}

\section{RESEARCH ARTICLE \\ 10.1029/2021SW002802 \\ Key Points: \\ - Solar wind speed corotation forecast error is affected by solar activity and spacecraft longitudinal and latitudinal separation \\ - Latitudinal separation has little effect when it is below $6^{\circ}$, but increasing importance above this \\ - A period in solar minimum gives a $46 \%$ increase in mean absolute error (MAE) from low to high latitudinal offset for Solar Terrestrial Relations Observatory (STEREO)-A and B corotations}

Supporting Information:

Supporting Information may be found in the online version of this article.

\section{Correspondence to:}

H. Turner,

h.turner3@pgr.reading.ac.uk

Citation:

Turner, H., Owens, M. J., Lang, M. S., \& Gonzi, S. (2021). The influence of spacecraft latitudinal offset on the accuracy of corotation forecasts. Space Weather, 19, e2021SW002802. https:// doi.org/10.1029/2021SW002802

Received 18 MAY 2021 Accepted 22 JUL 2021

\section{The Influence of Spacecraft Latitudinal Offset on the Accuracy of Corotation Forecasts}

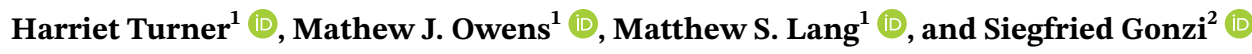 \\ ${ }^{1}$ Department of Meteorology, University of Reading, Reading, UK, ${ }^{2}$ Met Office, Exeter, UK
}

Abstract Knowledge of the ambient solar wind is important for accurate space weather forecasting. A simple-but-effective method of forecasting near-Earth solar wind speed is "corotation," wherein solar wind structure is assumed to be fixed in the reference frame rotating with the Sun. Under this approximation, observations at a source spacecraft can be rotated to a target location, such as Earth. Forecast accuracy depends upon the rate of solar wind evolution, longitudinal and latitudinal separation between the source and target, and latitudinal structure in the solar wind itself. The time-evolution rate and latitudinal structure of the solar wind are both strongly influenced by the solar cycle, though in opposing ways. A latitudinal separation (offset) between source and target spacecraft is typically present, introducing an error to corotation forecasts. In this study, we use observations from the Solar Terrestrial Relations Observatory (STEREO) and near-Earth spacecraft to quantify the latitudinal error. Aliasing between the solar cycle and STEREO orbits means that individual contributions to the forecast error are difficult to isolate. However, by considering an 18-month interval near the end of solar minimum, we find that the latitudinal-offset contribution to corotation forecast error cannot be directly detected for offsets $<6^{\circ}$, but is increasingly important as offsets increase. This result can be used to improve solar wind data assimilation, allowing representivity errors in solar wind observations to be correctly specified. Furthermore, as the maximum latitudinal offset between L5 and Earth is $\approx 5^{\circ}$, corotation forecasts from a future L5 spacecraft should not be greatly affected by latitudinal offset.

Plain Language Summary Space weather can damage our technologies, from power lines to satellites, as well as pose a hazard to humans in space. Forecasting space weather requires prediction of solar wind, a continuous outflow of material from the Sun, conditions in near-Earth space. A simple way to achieve this is "corotation," which assumes the structure of the solar wind is unchanging, but simply rotates around with the Sun. Thus, solar wind which sweeps past one spacecraft will arrive at Earth some time later. This forecast will be less accurate when the spacecraft is far from Earth, as we need to wait longer for the solar wind to rotate around, during which time its structure may have changed. If the spacecraft is at a different latitude to Earth, it will also create problems for the forecast. In this study, we quantify the contribution of these factors to the forecast error. This knowledge will improve corotation forecasts, but also aid in other, more sophisticated, forecast techniques. By defining how close spacecraft need to be to Earth to sample the same solar wind, it also helps define where future space weather monitoring spacecraft should be positioned.

\section{Introduction}

Space weather has the potential to damage electricity grids, cause satellite failures, disrupt communications, and threaten the health of humans in space (Cannon, 2013). The most severe space weather events are driven by transient coronal mass ejections (CMEs; Gosling, 1993). However, prediction of the ambient solar wind is still important for space weather for two reasons. First, the structure of the solar wind impacts the evolution of CMEs through interplanetary space and can determine their arrival time and severity at Earth (Case et al., 2008). Second, steady-state structures in the solar wind can also be a driver for space weather events in their own right (e.g., Alves et al., 2006). High-speed streams emanating from coronal holes can compress into the slower solar wind and form stream interaction regions (SIRs), which become corotating interaction regions (CIRs) if they persist for several solar rotations (Reiss et al., 2016). These are regions of higher plasma density and magnetic field strength and can cause geomagnetic disturbances 

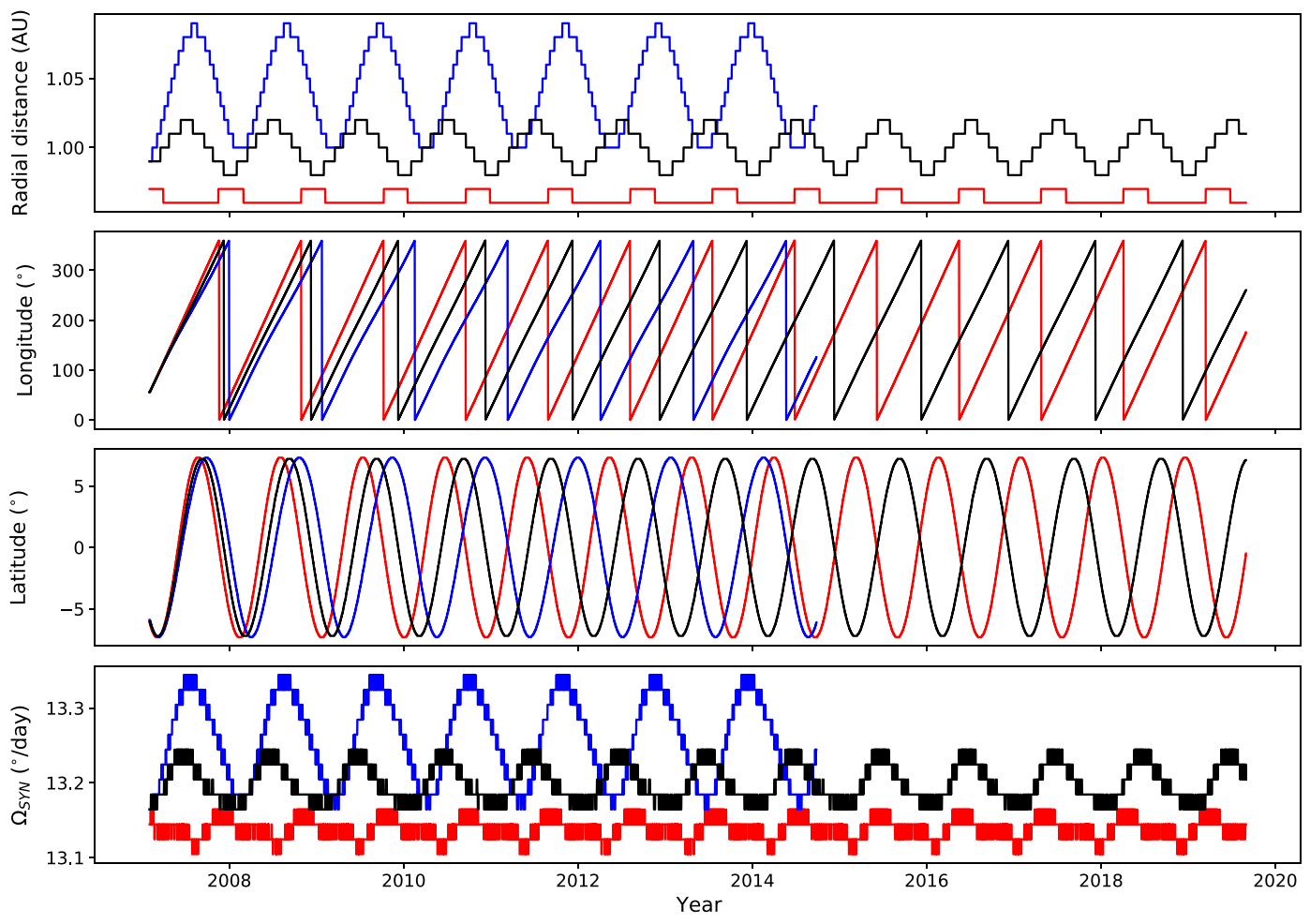

— STEREO-A — STEREO-B — Earth

Figure 1. Variation of radial distance, heliographic longitude, heliographic latitude, and synodic angular speed of Solar Terrestrial Relations Observatory (STEREO)-A (red), STEREO-B (blue), and Earth (black).

at Earth (Richardson \& Cane, 2012). Thus, for accurate space weather forecasting of both recurrent and non-recurrent events, knowledge of the solar wind conditions near-Earth is required.

The Sun's magnetic field forms an Archimedean spiral due to the field lines remaining rooted at the Sun as it rotates; this shape is known as the "Parker Spiral" (Parker, 1958). The solar wind flows almost radially away from the Sun; however, fast and slow structures rotate with the Sun, which has a rotational period of 27 days with respect to Earth. Assuming a steady-state solar wind structure in the rotating solar frame, this means that the same solar wind conditions will reoccur every 27 days at Earth. Owens et al. (2013) quantified the potential benefit of such 27-day "recurrence" (also called "27-day persistence") forecasts. They can provide useful long-lead time forecasts and a benchmark for more sophisticated models. The same principle can be used with observations from spacecraft distant from Earth, but at the same heliographic latitude, for example, on the ecliptic plane. Applying a time shift to observations at one spacecraft allows them to be used as a forecast for another, further on in the Sun's rotation. These are known as corotation forecasts and the reduction in forecast lead time limits (but does not eliminate) the assumption of a completely steady-state solar wind structure. They have been shown to be a useful forecasting tool, often outperforming the 27-day recurrence model (Bailey et al., 2020; Kohutova et al., 2016; Simunac et al., 2009; Thomas et al., 2018). For observations at the same heliocentric distance, the required time shift $(\Delta t)$ is given solely by corotation time $\left(t_{C}\right)$, the time it takes the Sun to rotate by the angle $\phi$ between the longitudes of the observation (referred to as the source) and the location to be forecast (referred to as the target). For $\phi=1^{\circ}, t_{C}=1.8 \mathrm{~h}$. However, spacecraft (and the Earth) orbiting the Sun typically have elliptical orbits, resulting in radial distance variations, as shown in panel 1 in Figure 1. This must also be accounted for in the calculation of $\Delta t$.

Due for launch in 2027, the European Space Agency (ESA) propose to place a space weather monitor at the L5 Lagrangian point, a gravitational null located $60^{\circ}$ behind Earth (Davies, 2020). This point would provide a view of the Sun-Earth line, and so could give a side-on view of Earth directed CMEs (Akioka et al., 2005). It would also present the opportunity to use corotation forecasts from L5 to predict the solar 
wind conditions near-Earth with a lead time of approximately 4.5 days. The accuracy of corotation forecasts from L5 has been investigated using data from the Solar Terrestrial Relations Observatory (STEREO; Kaiser et al., 2008) mission during specific phases of the operational lifetime (Bailey et al., 2020; Kohutova et al., 2016; Simunac et al., 2009; Thomas et al., 2018). The STEREO mission consisted of two spacecraft, STEREO-A ahead of Earth's orbit and STEREO-B behind, moving away from Earth at a rate of $\sim 22.5^{\circ}$ per year (Kaiser et al., 2008). Combining these observations with near-Earth data from the Advanced Composition Explorer (ACE; Stone et al., 1998) or the OMNI data set (Vokhmyanin et al., 2019) provides a number of periods where there are two spacecraft $60^{\circ}$ apart in longitude. Simunac et al. (2009) demonstrated that the profiles of solar wind speed are similar when using STEREO-B as a forecast for STEREO-A at $\phi \approx 60^{\circ}$ in July 2008. Further to this, Kohutova et al. (2016) found that using corotation from STEREO-B to ACE improved the forecast of the $B_{z}$ component of the interplanetary magnetic field when compared to a 27-day recurrence forecast. Thomas et al. (2018) used different combinations of STEREO and ACE to show the effectiveness of an L5 monitor. It was found that a number of solar wind parameters, including speed, density, and temperature, were well predicted using four combinations of spacecraft, with $\phi \approx 60^{\circ}$, to produce corotation forecasts. The geomagnetic storm time index (Dst) has also been effectively forecasted through corotation from STEREO-B to OMNI, when $\phi \approx 60^{\circ}$ (Bailey et al., 2020). In both cases, corotation provides an improvement from 27-day recurrence.

Although extensive research has been conducted on the effectiveness of corotation from L5, previous studies have been limited to short periods of time when the spacecraft are separated by $60^{\circ}$. The majority of previous analysis has been around periods of low solar activity (solar minimum). The corotation forecasting method assumes a steady-state solar wind; however, in reality the solar wind varies with time and the rate of evolution is linked to the 11-year solar cycle. At solar minimum, the steady-state assumption is more valid as solar wind structure slowly evolves with time. Conversely, at solar maximum the higher activity levels and rapid evolution of solar wind structure lead to the steady Sun assumption breaking down more readily (Owens et al., 2013). This means that longer corotation times, and therefore, longer forecast lead times are generally expected to be more accurate at solar minimum than at solar maximum.

As the Sun progresses through the solar cycle, the latitudinal structure of the solar wind changes. At solar minimum, there is a slow solar wind band centered on the heliographic equator, with faster winds emanating from coronal holes at higher latitudes (McComas et al., 2003). This latitudinal ordering breaks down at solar maximum, due to the weaker dipole component of the Sun's magnetic field (Wang \& Sheeley, 1991). The variation in latitudinal structure is important for corotation forecasts, as spacecraft in the ecliptic plane vary in heliographic latitude $(\theta)$ owing to the $7.25^{\circ}$ tilt between the ecliptic plane and the rotational plane of the Sun, as shown in panel 3 of Figure 1. This results in a "latitudinal offset" $(\Delta \theta)$ between the point of observation (the source) and the location where the forecast is required (the target). The term "latitudinal offset" is used here as the latitudinal separation of the source and target spacecraft. This can introduce a representation error into corotation forecasts that needs to be accounted for in solar wind data assimilation (DA; Owens et al., 2020).

As will be demonstrated below, the available observations from the STEREO mission make the effect of $\Delta \theta$ on solar wind speed corotation forecast accuracy difficult to disentangle from solar activity and $\Delta t$. In particular, the contribution of latitudinal offset errors to the total corotation forecast error is expected to be significantly higher at solar minimum than solar maximum. Conversely, the contribution from $\Delta t$ will increase as the corona becomes more dynamic, which is at solar maximum. Finally, as all spacecraft are in the ecliptic plane, large latitudinal spacecraft separation can only occur when there is also large longitudinal separation (and hence large $\Delta t$ ). Thus, all contributions to corotation forecast error are interdependent.

Previous study into the error introduced by $\Delta \theta$ in corotation forecasts has been investigated using steadystate model output. Owens et al. $(2019,2020)$ showed that $\Delta \theta$ can have a significant effect on corotation forecast error, especially at solar minimum. It was found that during solar minimum, due to the band of slow solar wind near the heliographic equator, the solar wind could be considered broadly similar up to $|\Delta \theta|=3^{\circ}$. Although this increases to $|\Delta \theta|=10^{\circ}$ during solar maximum, there are increasing numbers of transient events and smaller-scale turbulence (Owens et al., 2020). This shows a breakdown of the latitudinal structure found at solar minimum. Corotation forecast error from L5 to Earth was shown to be up to $80 \mathrm{~km} \mathrm{~s}^{-1}$ at solar minimum purely due to $\Delta \theta$ (Owens et al., 2019), though the peak value occurs around the 
winter and summer solstices. Averaged over the year, this reduces to around $50 \mathrm{~km} \mathrm{~s}^{-1}$. Although models have shown that a strong effect of $\Delta \theta$ on wind speed corotation forecast error is expected, Allen et al. (2020) found that the arrival time of SIR/CIRs does not seem to be affected. This may be the result of compensating errors, as shown below.

The Owens et al. $(2019,2020)$ work was motivated by improving solar wind DA capabilities (Lang et al., 2017, 2021). DA combines model output and observational data to find an optimal estimation of reality and is beginning to be used in solar wind forecasting (Lang \& Owens, 2019). Quantifying the error from $\Delta \theta$ allows observations to be fully utilized through the specification of more accurate observation error covariances and the removal of any potential biases. Observation errors can arise through poor specification of the observations position, resolution difference between observations and the model, pre-processing errors and many others. These are known as representivity errors, as they are errors that arise from poor representation of the observations, and they are what we wish to specify with greater accuracy in solar wind DA. The interested reader can find more information on representivity errors in Janjić et al. (2018). DA is a step forward in the use of observations for solar wind forecasting as it allows for the observations to be mapped to all longitudes and radial distances, whereas corotation only gives a forecast for a single point. Current DA schemes developed for solar wind forecasting make use of solar wind speed observations (Lang \& Owens, 2019; Lang et al., 2017, 2021). Although both corotation and DA can be used for forecasting parameters such as plasma density and magnetic polarity, at present, the DA methods presented in Lang and Owens (2019) and Lang et al. (2017) only use solar wind speed. Therefore, this is the parameter investigated in this study to aid comparison with DA results. Furthermore, the flow dominated regime of the solar wind, coupled with the "frozen-in" flux theorem indicates that if the solar wind speed is not correctly forecast, timing errors will be present in the forecast of other solar wind parameters, such as solar wind density and heliospheric magnetic field.

Due to the previous reliance on model output for analysis of the effect of $\Delta \theta$ on corotation forecast error, it is necessary to investigate whether this is present in observational solar wind data. Magnetohydrodynamic (MHD) models produce a solar wind that is "smoother" than what is observed. This means that an error introduced from $\Delta \theta$ could be more easily detected as there are no transient events or any small-scale turbulence. Furthermore, analysis of the model output was steady state, and so no time variation of the solar wind was captured. This study uses solar wind data from the STEREO mission and near-Earth to investigate the error introduced from $\Delta \theta$.

Sections 2 and 3 will detail the data and methods used to produce our analysis, followed by the results in Section 4. Finally, we will discuss and draw conclusions in Section 5.

\section{Data}

Corotation forecasts require spacecraft at approximately the same heliocentric distance $(R)$ as the intended forecast position (typically Earth). Although radial scaling from Parker Solar Probe to 1 AU has been successfully used for high-speed streams (e.g., Allen et al., 2021; Perrone et al., 2019; Wijsen et al., 2021), here, we use observations from 1 AU. The solar wind captured in this study covers a broad range of solar wind speeds and so as it evolves radially, solar wind streams will interact, and this may not be captured when scaling. Spacecraft at approximately the same heliographic latitude $(\theta)$ are also required, but they must be separated in heliographic longitude $(\phi)$. Greater $\phi$ implies longer forecast lead times, but also decreases the forecast reliability, as will be demonstrated. Furthermore, throughout this analysis, only the radial component of the solar wind is used to allow direct comparison between observation sources.

The twin STEREO (Kaiser et al., 2008) spacecraft, in conjunction with near-Earth observations from the OMNI data set (Vokhmyanin et al., 2019), provide a unique opportunity to test corotation forecasting and to better understand the factors that contribute to errors. Here, we use 1-h STEREO plasma data obtained from the plasma and suprathermal ion composition (PLASTIC) instrument (Galvin et al., 2008) and downloaded from CDAWeb (cdaweb.gsfc.nasa.gov). One-hour OMNI data are obtained from OMNIWeb (omniweb.gsfc. nasa.gov). The heliographic locations of STEREO spacecraft and Earth were obtained from OMNIWeb (omniweb.gsfc.nasa.gov/coho/helios/heli.html). We additionally use daily sunspot number from Sunspot Index and Long-term Solar Observations (SILSO) as a proxy for solar activity, downloaded from sidc.be/silso. 
Table 1

The Possible Corotation Forecast Configurations Using STEREO-A, STEREO-B, and OMNI Solar Wind Data, Where the Data From the Source Spacecraft is Used as a Forecast for the Target Spacecraft

\begin{tabular}{lcc}
\hline Source spacecraft & Target spacecraft & Time period \\
\hline STEREO-A & OMNI & Feb 2007-Aug 2019 \\
STEREO-A & STEREO-B & Feb 2007-Aug 2014 \\
STEREO-B & OMNI & Feb 2007-Aug 2014 \\
STEREO-B & STEREO-A & Feb 2007-Aug 2014 \\
OMNI & STEREO-A & Feb 2007-Aug 2019 \\
OMNI & STEREO-B & Feb 2007-Aug 2014 \\
\hline
\end{tabular}

Note. A gap in the STEREO-A data exists between August 2014 and November 2015, affecting the STEREO-A to OMNI and OMNI to STEREO-A corotations.

Abbreviation: STEREO, Solar Terrestrial Relations Observatory.
The STEREO spacecraft separate from Earth at a rate of $\approx 22.5^{\circ}$ per year. Thus, from launch in late 2006, they passed behind the Sun in 2014 leading to a data gap in the STEREO-A data from August 2014 to November 2015 and the loss of communication with STEREO-B from August 2014. While both spacecraft orbit the Sun at a distance close to $1 \mathrm{AU}, R$ varies by up to $0.11 \mathrm{AU}$ and must be accounted for when computing corotation forecasts. For completeness, we also take account of Earth's orbital eccentricity, though the associated change in $R$ is small ( $\sim 0.01 \mathrm{AU})$.

Here, OMNI data are used for near-Earth solar wind observations. Solar wind data from a succession of spacecraft (including WIND and ACE) located at the L1 Lagrange point is propagated to the bowshock of Earth, providing another source of data for use in corotation forecasts alongside the STEREO spacecraft (Vokhmyanin et al., 2019).

A single spacecraft can be used to provide a corotation forecast for one whole Carrington rotation (approximately 27 days) ahead. Such 27-day recurrence forecasts have already been considered in detail (Owens et al., 2013). The maximum latitude difference for a single in-ecliptic spacecraft over a 27-day period occurs at the equinoxes and reaches a magnitude of approximately $3.5^{\circ}$. As will be demonstrated later (see Figure 6), this is insufficient to quantify the latitudinal-offset contribution to corotation forecast error. Thus, we focus on pairs of spacecraft. By combining the STEREO-A, B, and OMNI spacecraft, there are six potential pairs of source (at the position where the solar wind observations are made) and target (at the position of the forecast) spacecraft. These can be seen in Table 1 .

\section{Methods}

Solar wind speed corotation forecasts are calculated and tested from combinations of the STEREO and OMNI spacecraft observations of solar wind speed $(V)$. Throughout this analysis, solar wind speed is the only quantity forecast and the radial component is used. Simunac et al. (2009) and Thomas et al. (2018) describe this process in terms of mapping between Carrington longitudes at pairs of spacecraft. We, here, use an equivalent description in terms of time, to make explicit a number of assumptions.

Each hourly $V$ observation at the source spacecraft $\left(V_{S}\right)$ is used to produce a forecast $\left(V_{F}\right)$ at the target spacecraft location at a time $\Delta t$ in the future:

$$
V_{F}(t+\Delta t)=V_{S}(t)
$$

where $V_{S}(t)$ is the observed solar wind speed at the source spacecraft at time $t$. $\Delta t$ is the required time delay for the same solar wind observed at the source location to reach the target location. Consequently, it is also the forecast lead time. $\Delta t$ consists of two elements: $t_{R}$, the time for solar wind to propagate between the source and target radial distances $\left(R_{S}\right.$ and $R_{T}$, respectively) and $t_{C}$, the time for solar wind sources to rotate between the source and target longitudes, accounting for spacecraft motion in the inertial frame. This is shown schematically in Figure 2.

Figure 2a shows a time $t=t_{0}$, when the target spacecraft has a longitude of $\phi_{T 0}$ and a radial distance of $R_{T}$. Similarly, the source spacecraft has a longitude of $\phi_{S 0}$ and a radial distance of $R_{S}$. The first step is to ballistically map the source observations to a radial distance of $R_{T}$ :

$$
t_{R}=\frac{R_{T}-R_{S}}{V_{S}\left(t_{0}\right)}
$$

where $t_{R}$ is the radial propagation time. As the propagation in radial distance is purely ballistic, it ignores any stream interaction effects or solar wind acceleration. Thus this approach is only valid for $\left|R_{T}-R_{S}\right| \ll R_{T}$. Given the radial variations of the spacecraft are slow, we assume $R_{S}$ and $R_{T}$ are constant over the interval $\Delta t$. As Figure 1 shows, the spacecraft can be separated by up to approximately $0.1 \mathrm{AU}$ in radius. Therefore, depending on the solar wind speed $V_{S}$, the radial propagation time can range from 6 to $14 \mathrm{~h}$ (for solar wind 
(a) Time $t=t_{0}$

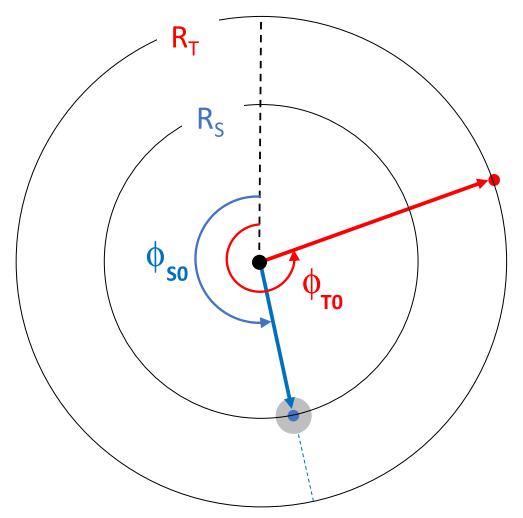

(b) Time $t=t_{0}+t_{R}$

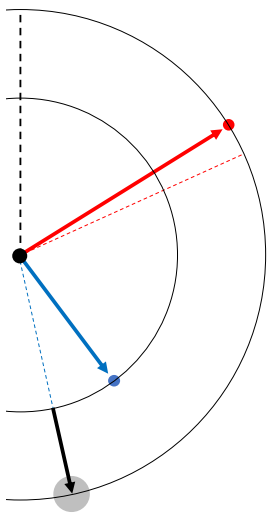

(c) Time $t=t_{0}+t_{R}+t_{C}$

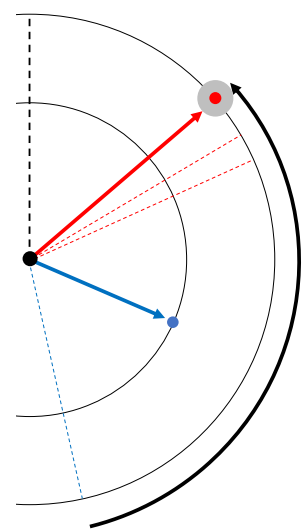

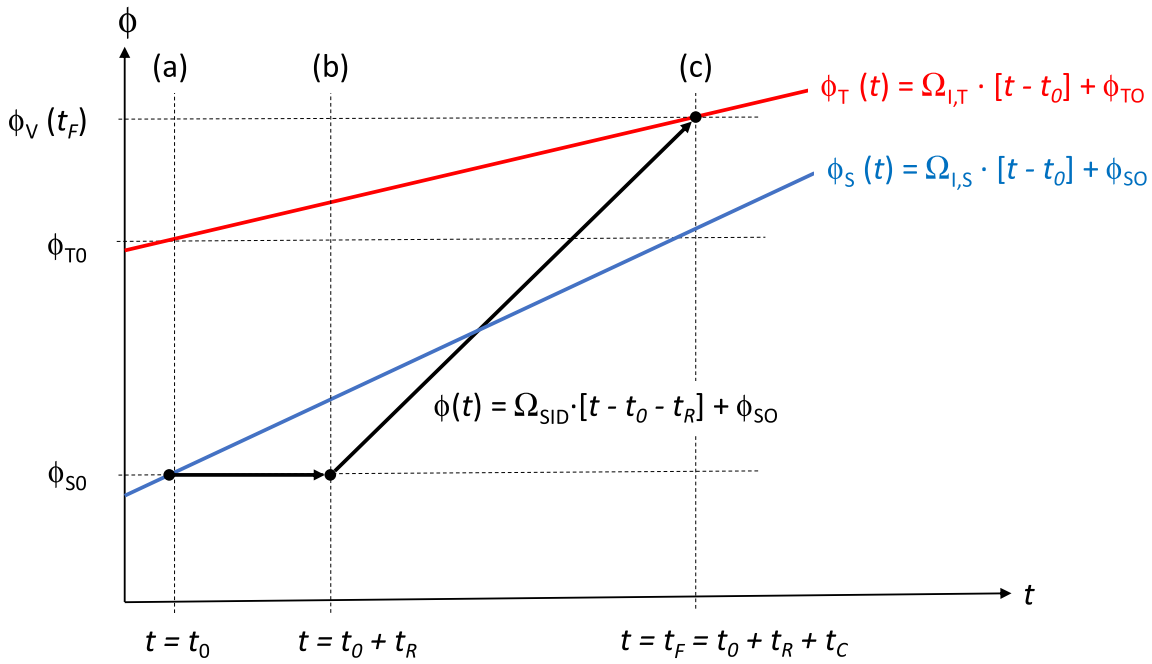

Figure 2. A schematic of the corotation forecast in the inertial frame. The gray circle tracks the position of a solar wind structure observed by the source spacecraft at time $t=t_{0}$. Top: (a) At time $t=t_{0}$, the target spacecraft (red dot) has a longitude of $\phi_{T 0}$ and radial distance of $R_{T}$, while the source spacecraft (blue dot) is at $\phi_{S 0}$ and $R_{S}$. (b) Radial propagation from $R_{S}$ to $R_{T}$ takes a time $t_{R}(\mathrm{c})$ Corotation from $\phi_{S 0}$ to the target spacecraft takes time $t_{C}$. Bottom: The same steps shown as a time series of $\phi . \Omega_{I, S}$ and $\Omega_{I, T}$ denotes the orbital angular speed of the source and target spacecraft, respectively, while $\Omega_{S I D}$ denotes the sidereal rotation speed of the Sun.

speeds of 700 and $300 \mathrm{kms}^{-1}$, respectively). This can be a significant contribution to $\Delta t$ and therefore it is important to include.

Figure $2 \mathrm{~b}$ shows that during the interval $t_{R}$, the target spacecraft continues to move ahead in longitude at an orbital angular speed in the inertial frame of $\Omega_{I, T}$. Note that if $R_{S}>R_{T}, t_{R}$ will be negative and spacecraft will move to smaller $\phi$ during radial propagation. STEREO-A, B, and OMNI spacecraft have different average values of $\Omega_{I}$, which allows the spacecraft to separate over time. For all three spacecraft these values also vary slowly over the year, owing to the slightly elliptical orbits. This is shown in panel 4 of Figure 1 . We account for this effect by computing $\Omega_{I}$ from the change in $\phi$ over a 5 -day window centered on time $t_{0}$. (This window is short enough to allow for the change in $\Omega_{I, F}$ over the year, but large enough to remove numerical noise from taking the time gradient of $\phi_{V}$ ). Over the interval $\Delta t$, which is typically a few days, it is reasonable to assume a constant value of $\Omega_{I, S}$, which allows us to express the longitude of the target spacecraft as:

$$
\phi_{T}\left(t_{0}\right)=\Omega_{I, T} \cdot t_{0}+\phi_{T 0}
$$



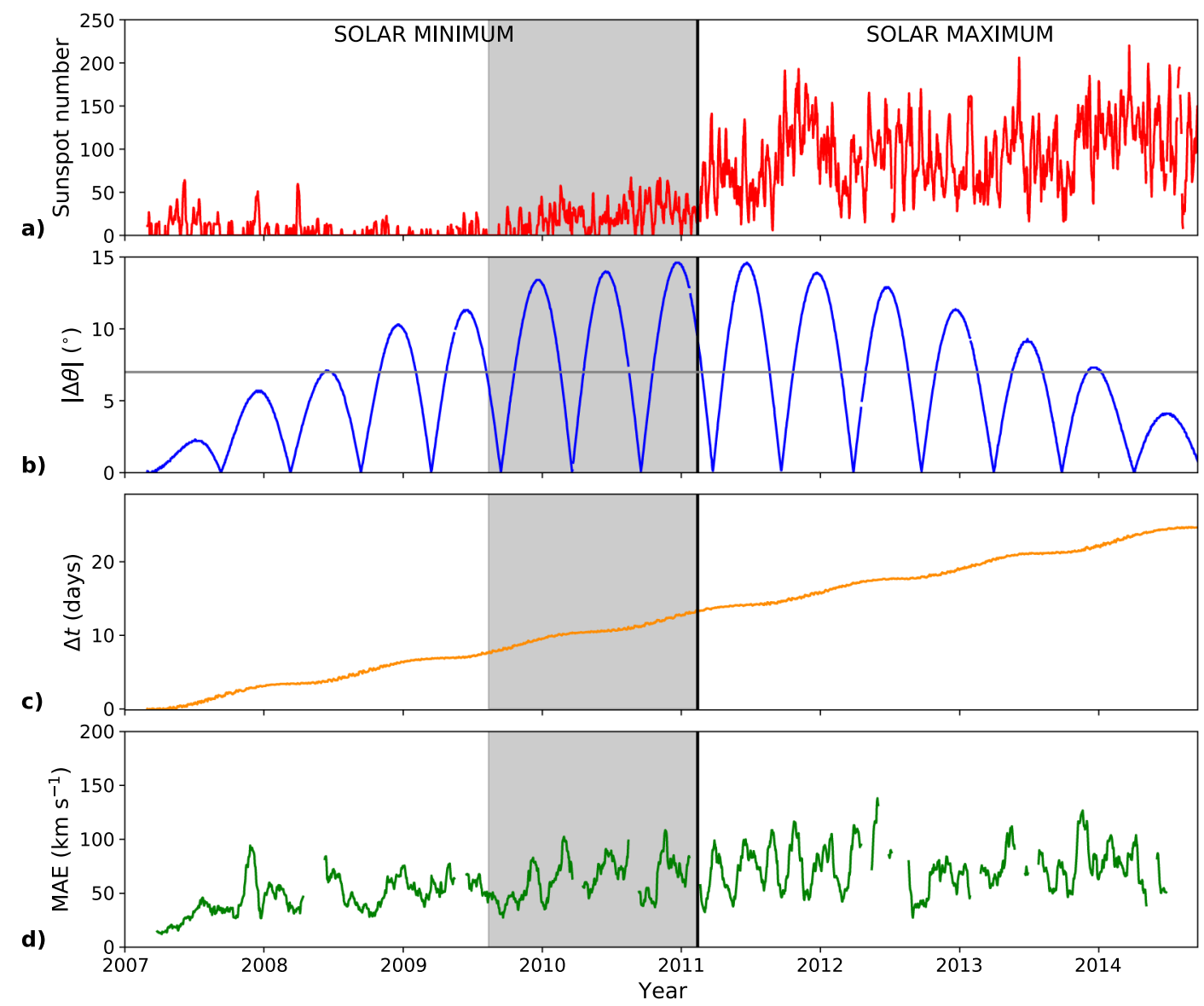

Figure 3. Time series at daily resolution for the Solar Terrestrial Relations Observatory (STEREO)-B to A corotation forecast, covering the duration of the STEREO-B lifetime. Here, STEREO-B is the source spacecraft and STEREO-A is the target spacecraft. (a) Sunspot number. (b) $|\Delta \theta|$, the absolute latitude difference between the source and target location. The gray horizontal line indicates $7^{\circ}$, the separation between high and low $|\Delta \theta|$ used in this study. (c) $\Delta t$, the forecast lead time. (d) 27-day rolling average of the daily mean absolute error (MAE) for the corotation forecast. In all panels, the vertical black line separates the definitions of solar minimum and solar maximum used in this study (see main text). The gray-shaded region highlights an interval during solar minimum used for further investigation.

After a time $t_{R}$, the solar wind structure observed by the source spacecraft at $t_{0}=0$ will corotate with the solar wind, meaning its longitude varies as:

$$
\phi\left(t_{0}\right)=\Omega_{S I D} \cdot\left[t_{0}-t_{R}\right]+\phi_{S 0}
$$

where $\Omega_{S I D}$ is the sidereal rotation speed of the Sun (i.e., $2.86 \times 10^{-6} \mathrm{rad} \mathrm{s}^{-1}$ ). Thus, it will encounter the target spacecraft at a time $\Delta t$, where:

$$
\Delta t=\frac{1}{\Omega_{S Y N, T}}\left[\phi_{T 0}-\phi_{S 0}+\Omega_{S I D} \cdot t_{R}\right]
$$

where $\Omega_{\mathrm{SYN}, T}$ is the synodic orbital angular speed of the target spacecraft, given by $\Omega_{\mathrm{SYN}, T}=\Omega_{S I D}-\Omega_{I, T}$.

For the STEREO-B corotation forecast of $V$ at STEREO-A's position, $\Delta t$ is shown as the orange line in Figure 3c.

The observed radial solar wind speeds at the target and source spacecraft $\left(V_{T}\right.$ and $V_{S}$, respectively) are taken from 1-h resolution data. While the forecast speed, $V_{F}$, is computed from hourly $V_{S}$ data, the $t_{R}$ term means that the computed forecast speed, $V_{F}$, is no longer on a regular 1-h time step. Thus, $V_{F}$ is linearly interpolated back to a standard hourly time base for direct comparison with the $V_{T}$. 


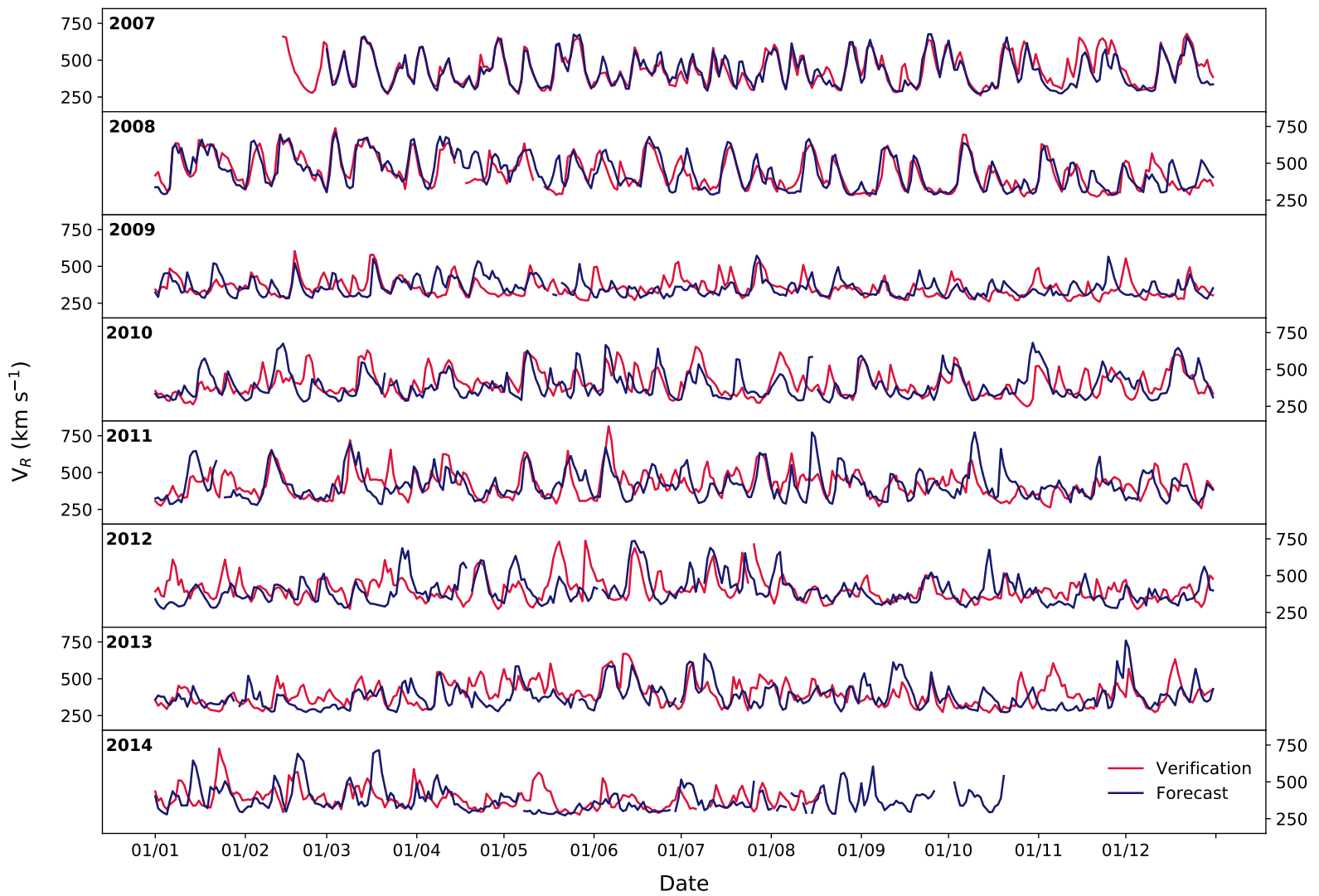

Figure 4. Time series of solar wind speed at Solar Terrestrial Relations Observatory (STEREO)-A's location. In red is the observed solar wind speed by STEREO-A. In blue is the forecast solar wind speed, $V_{F}$, produced from time shifting the STEREO-B observations. Thus, STEREO-A is the target spacecraft providing the observations to verify the forecast, and STEREO-B is the source spacecraft used to produce $V_{F}$. Data have been averaged to 1-day resolution for clarity.

Figure 4 shows a stack plot of $V$ at STEREO-A's location. This uses a forecasted solar wind from STEREO-B, $V_{F}$ in blue, with observations by STEREO-A, $V_{T}$ in red. $V_{F}$ is produced from time shifting the STEREO-B observations and $V_{T}$ is used to verify the forecast. Data have been further averaged to 1-day resolution for clarity. It can be seen that the agreement is extremely good for 2007 and 2008, and becomes gradually worse as time progresses. To quantify the degree of agreement we use the MAE between the observed and forecast $V$ :

$$
M A E=\frac{1}{N} \sum_{n=1}^{N}\left|V_{T}\left(t_{n}\right)-V_{F}\left(t_{n}\right)\right|
$$

where $N$ is the total number of time steps considered. MAE is a point-by-point analysis and thus small timing errors in the forecast can be heavily penalized (Owens, 2018). In the case of a corotation forecast, where the solar wind structure is assumed to be merely time lagged between two positions, the general solar wind structure should be well reproduced. Therefore, MAE is an appropriate metric to use as timing errors indicate a time evolution of the solar wind, for which corotation forecasts should be penalized.

The green line in Figure 3d shows 27-day averages of MAE for STEREO-B observations used to forecast the solar wind conditions at STEREO-A. The increase in MAE in 2007 through 2009 is consistent with the divergence in the time series seen in Figure 4. When using other corotations, such as STEREO-B to OMNI and OMNI to STEREO-A, for Figures 3 and 4, it is found that the plots are qualitatively similar. These are provided in the supporting information for the interested reader. In Section 4, we consider the different contributions to this MAE, with a focus on quantifying the role of latitudinal difference between the forecast and verification spacecraft. This is measured as:

$$
\Delta \theta(t)=\theta_{T}(t)-\theta_{S}(t-\Delta t)
$$




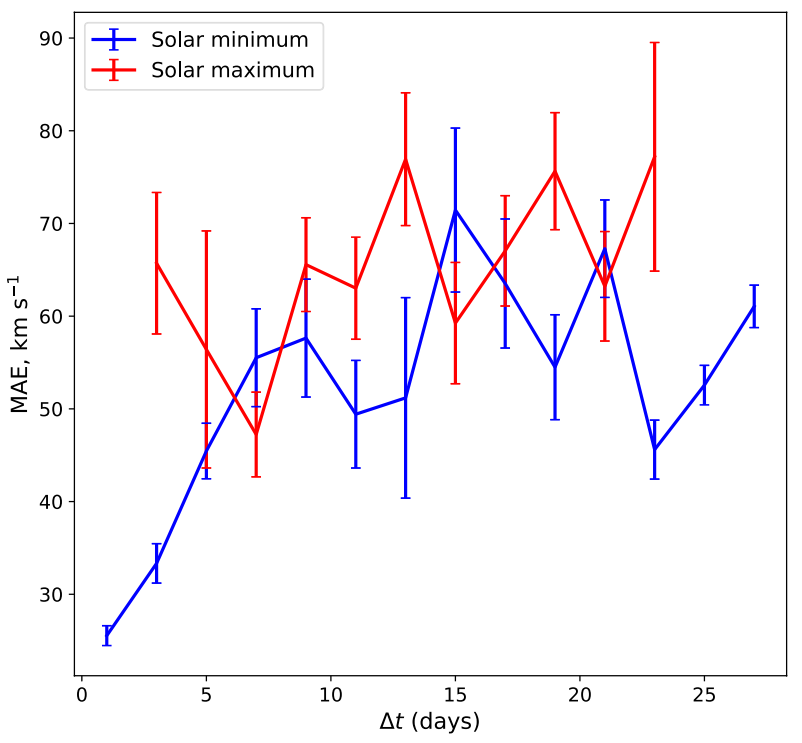

Figure 5. Forecast mean absolute error (MAE) as a function of forecast lead time, $\Delta t$, for solar minimum and maximum periods. Lines show the mean values, while error bars span one standard error on the mean. All Solar Terrestrial Relations Observatory (STEREO)/OMNI spacecraft pairings are used. Only periods with $|\Delta \theta|<2^{\circ}$ are included. where $\theta_{T}$ and $\theta_{S}$ are the heliographic latitudes of the target and source spacecraft/positions, respectively. The absolute value of $\Delta \theta(t)$ is shown in Figure 3b.

Combining the data from the six corotation configurations listed in Table 1 allows for the most robust analysis of the variables affecting the forecast error. There are, however, a number of biases in the sampling of this data set that make it complex to isolate individual factors in corotation forecast error. In particular, the motion of the STEREO spacecraft results in strong aliasing of the $|\Delta \theta|$ and $\Delta t$ with both each other and the solar cycle, and mean that confounding variables and compensating errors are an ever-present problem.

In order to coarsely isolate the influence of solar activity, the data are split into periods of solar minimum and maximum. Given only a single solar maximum is covered by the STEREO data set and the loss of communication with STEREO-B reducing it further, a simple sunspot number threshold is appropriate. We choose a value of 75 sunspots in the total daily sunspot number time series, as this selects the transition from solar minimum to solar maximum in February 2011, when there is a clear step-change in daily sunspot number. Using the same threshold puts the transition from solar maximum to minimum in March 2016.

We further split the data into periods of high and low $|\Delta \theta|$. Given the maximum $|\Delta \theta|$ available with the STEREO/OMNI data set is approximately $14.5^{\circ}$, we use a cut-off of $7^{\circ}$, though this will be further investigated in the remainder of the study.

\section{Results and Discussion}

We first consider the effect of forecast lead time, $\Delta t$, on forecast accuracy. To minimize the influence of $|\Delta \theta|$, we limit analysis to periods when $|\Delta \theta|<2^{\circ}$. Figure 5 shows forecast MAE as a function of $\Delta t$, for all spacecraft pairings. In general, MAE at solar maximum is higher than at solar minimum for the same $\Delta t$, as expected. At solar minimum, there is a trend for increasing MAE with increasing $\Delta t$ out to around 7 days. However, past 20 days, MAE decreases. $\Delta t>20$ days is confined exclusively to STEREO-A to STEREO-B forecasts very early in the mission, when the spacecraft were still near Earth. Thus, the reduced MAE may actually be the result of particularly quiet solar activity levels at this time. Despite 2007-2010 being classified as solar minimum on the basis of a sunspot number threshold, Figure 4 shows a clear difference in the character of the solar wind speed structures between 2007 and 2009, with recurrent fast streams giving way to more persistent slow wind.

Encapsulated within $\Delta t$ is the effect from radial separation. Analyzing this effect, limiting to times when $|\Delta \theta|<2^{\circ}$, it is found that there is so obvious trend between MAE and radial separation. This plot is included in the supporting information for the interested reader.

Next, we consider the effect of $|\Delta \theta|$. Figure 6 shows the differing latitudinal dependencies between solar minimum and solar maximum. At solar maximum, the MAE remains relatively constant at $\sim 80 \mathrm{~km} \mathrm{~s}^{-1}$. At solar minimum, there is a clear correlation between $|\Delta \theta|$ and MAE. However, it is here likely the result of aliasing of $|\Delta \theta|$ with $\Delta t$ and/or sunspot number. This is shown by Figure 7. During the solar minimum interval, splitting the data into high and low $|\Delta \theta|$ does not give equal sampling of either sunspot number or forecast lead time. Thus while MAE is lower for low $|\Delta \theta|$, we cannot rule out the lower mean sunspot number or the existence of lower $\Delta t$ values as being the causal effect.

For comparison, Figure 6 also shows the MAE as a function of $|\Delta \theta|$ from previous modeling studies (Owens et al., 2019, 2020). In those studies, the "Magnetohydrodynamics Algorithm outside a Sphere" (MAS) global coronal and heliosphere model (Linker et al., 1999; Riley et al., 2012) was constrained by the observed photospheric magnetic field and used to reconstruct the solar wind speed at $1 \mathrm{AU}$. As the solar wind solutions 

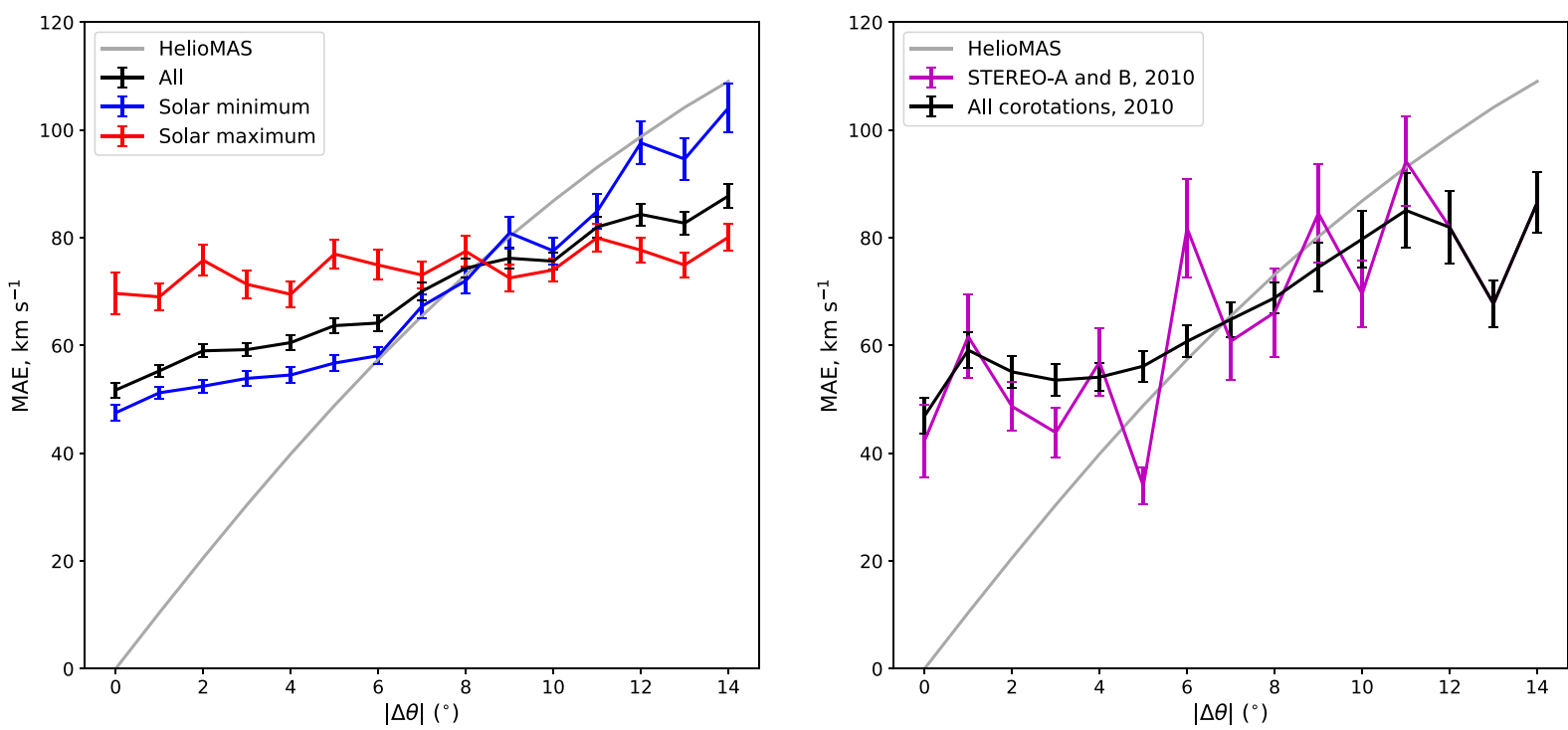

Figure 6. Variation of mean absolute error (MAE) with $|\Delta \theta|$. Unless otherwise stated, all spacecraft pairings are included. Lines show the mean values, while error bars span one standard error on the mean. Left: The entire data set (black), further split into solar maximum (red) and minimum (blue). While there appears to be a correlation between MAE and $|\Delta \theta|$ at solar minimum, this could be a result of aliasing of $|\Delta \theta|$ with $\Delta t$. Thus we also show (right) a combination of Solar Terrestrial Relations Observatory (STEREO)-A to B and STEREO-B to A corotations (magenta) and using all corotations (black) for a limited period from August 2009 to February 2011. Both panels show the equivalent modeled MAE at different $|\Delta \theta|$ for steady-state solar wind model solutions (gray). See body text for further description.

are steady state, the MAE obtained is purely a result of $|\Delta \theta|$ and should represent a lower limit to that expected observationally, where transient structures, small-scale turbulence and rapidly evolving large-scale structures providing additional sources of MAE for corotation forecasts. This agrees somewhat with our findings: At low $|\Delta \theta|$ values $\left(<7^{\circ}\right)$, the observed MAE is higher than the model result, suggesting that $|\Delta \theta|$ is not the dominant contribution to MAE in this parameter range. The model and observations approximately agree in the range $7^{\circ} \leq|\Delta \theta| \leq 11^{\circ}$, suggesting that $|\Delta \theta|$ has become a significant contribution to MAE. For $|\Delta \theta|>11^{\circ}$, the observational MAE is lower than the model result, suggesting that the difference in solar wind speed with latitude present in the models gets reduced by other processes.

In order to better isolate the effect of $|\Delta \theta|$ in our observational data set, it is necessary to further subdivide it. However, a competing requirement is to retain enough data for meaningful statistical analysis. A compromise of these factors is shown as the gray-shaded region in Figure 3, which spans August 2009 to February 2011. This provides a period of time where $|\Delta \theta|$ rises to a large enough value (maximum of $14.9^{\circ}$ ) but it is still relatively close to solar minimum, when the latitudinal effect is expected. High and low $|\Delta \theta|$ periods are approximately evenly spaced through this period, meaning both contain similar levels of solar activity. Finally, by comparing the STEREO-A to B forecasts with STEREO-B to A forecasts, we can effectively eliminate $\Delta t$ as contributing factor, as the two combinations have opposing $\Delta t$ trends.

As can be seen in Figure 8, the interval from August 2009 to February 2011 provides approximately equal sunspot number distributions for high and low $|\Delta \theta|$ periods (see also Table 2). $\Delta t$ distributions are also in approximate agreement, particularly when STEREO-A to B and STEREO-B to A forecasts are combined. Furthermore, the difference in the MAE histograms is consistent when the source/target spacecraft, and hence bias in $\Delta t$, are switched. Thus, we can reasonably conclude that the observed difference in MAE between high and low $|\Delta \theta|$ periods shown here is not the result of aliasing with other effects.

It is apparent from Table 2 that there is a distinct difference in the average corotation forecast MAE for high and low $|\Delta \theta|$ times. The average values for MAE are statistically distinct (i.e., differ by far more than one standard error on the mean). Low $|\Delta \theta|$ periods produce a significantly lower average corotation forecast MAE for both STEREO-B to A and A to B corotations, thus, $\Delta t$ is unlikely to be a factor. This is further seen by mean $\Delta t$ for the combined data set being the same for high and low $|\Delta \theta|$ times within uncertainties. The same result is found for sunspot number. 

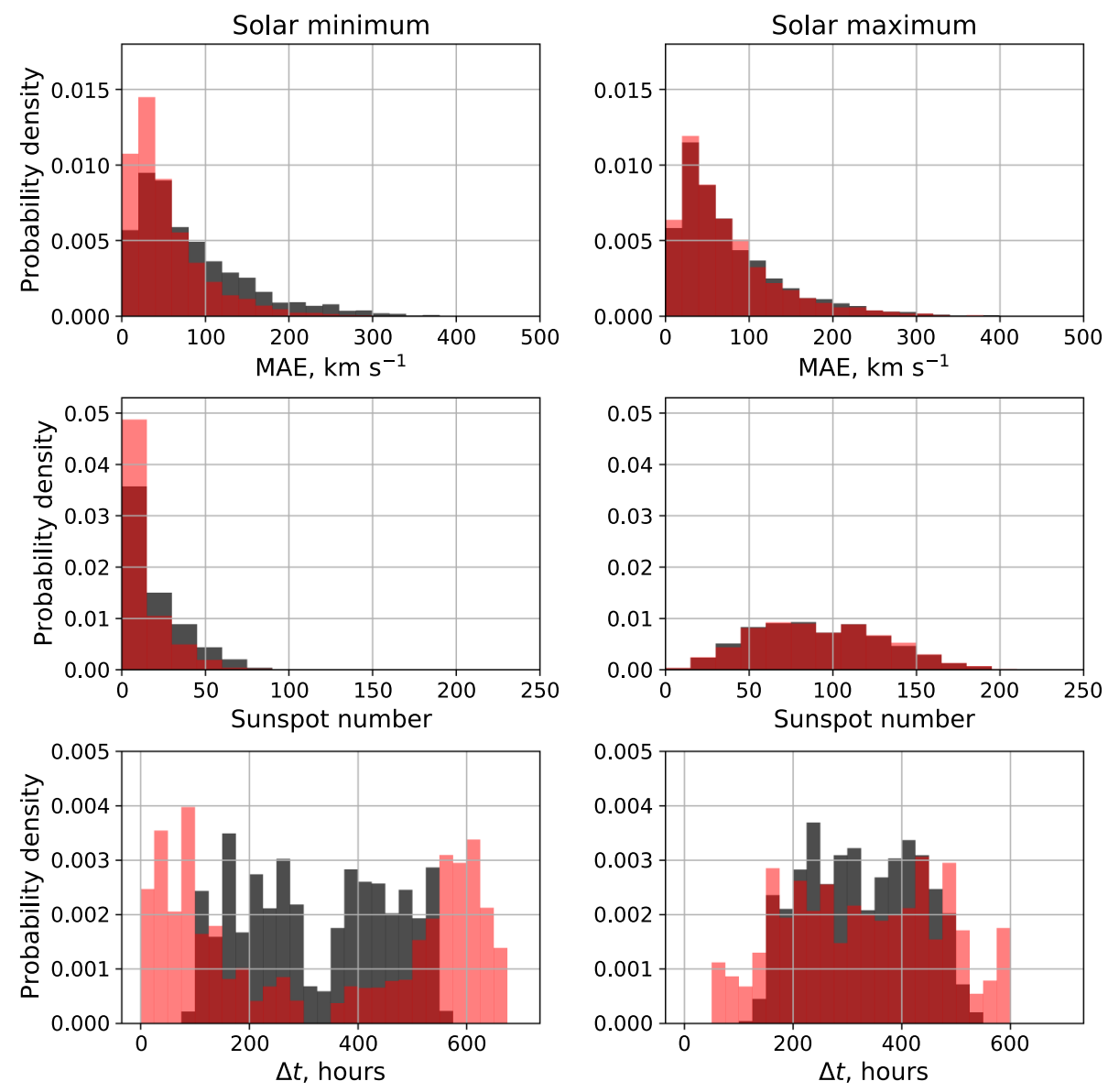

$\operatorname{High}|\Delta \theta|$

$\operatorname{Low}|\Delta \theta|$

Figure 7. Probability density of (top) the mean absolute error (MAE) between the observed and forecast $V$, (middle) sunspot number and (bottom) $\Delta t$. Data have been split into solar minimum (left) and solar maximum (right), and into high (black) and low (red) $|\Delta \theta|$ using a threshold of $7^{\circ}$. All Solar Terrestrial Relations Observatory (STEREO)/OMNI spacecraft pairings are included. The dark red areas show where there is overlap between high and low $|\Delta \theta|$.

Further to this, Table 3 shows the percentage increase in MAE, sunspot number, and $\Delta t$ from low to high $|\Delta \theta|$ averages in Table 2. Percentage difference is calculated as:

$$
\% \text { difference }=\frac{x_{\text {high }}-x_{\text {low }}}{x_{\text {low }}} \times 100
$$

where $x_{\text {high }}$ and $x_{\text {low }}$ is the data at high and low $|\Delta \theta|$, respectively.

The percentage increase in MAE is consistent at around $46 \%$ for the two corotations and when both data sets are combined. It is slightly lower when including the OMNI data, as this skews the data set in favor of low $|\Delta \theta|$ occurrence. For sunspot number, we can see that there is very little increase from low to high $|\Delta \theta|$, with the percentage increases below $2 \%$ for the STEREO-A and B corotations. Although there is a positive and negative percentage increase between $\Delta t$ from low to high $|\Delta \theta|$, this seems to have little impact on the difference for MAE. As Figure 5 shows, for solar minimum, the effect of corotation time on MAE increases to $\Delta t=\sim 7$ days $(168 \mathrm{~h})$, where it remains approximately constant thereafter. This could explain the minimal effect that differing $\Delta t$ has for high and low $|\Delta \theta|$, as both for both cases, $\Delta t>8$ days.

Comparing these results with the right-hand panel of Figure 6, we can see that the MAE contribution from $|\Delta \theta|<6$ remains approximately constant, before increasing. To assess this, correlation coefficients of MAE and $|\Delta \theta|$ were calculated for $|\Delta \theta|<6$ and $|\Delta \theta| \geq 6$. These are shown in Table 4 . We can see the transition 

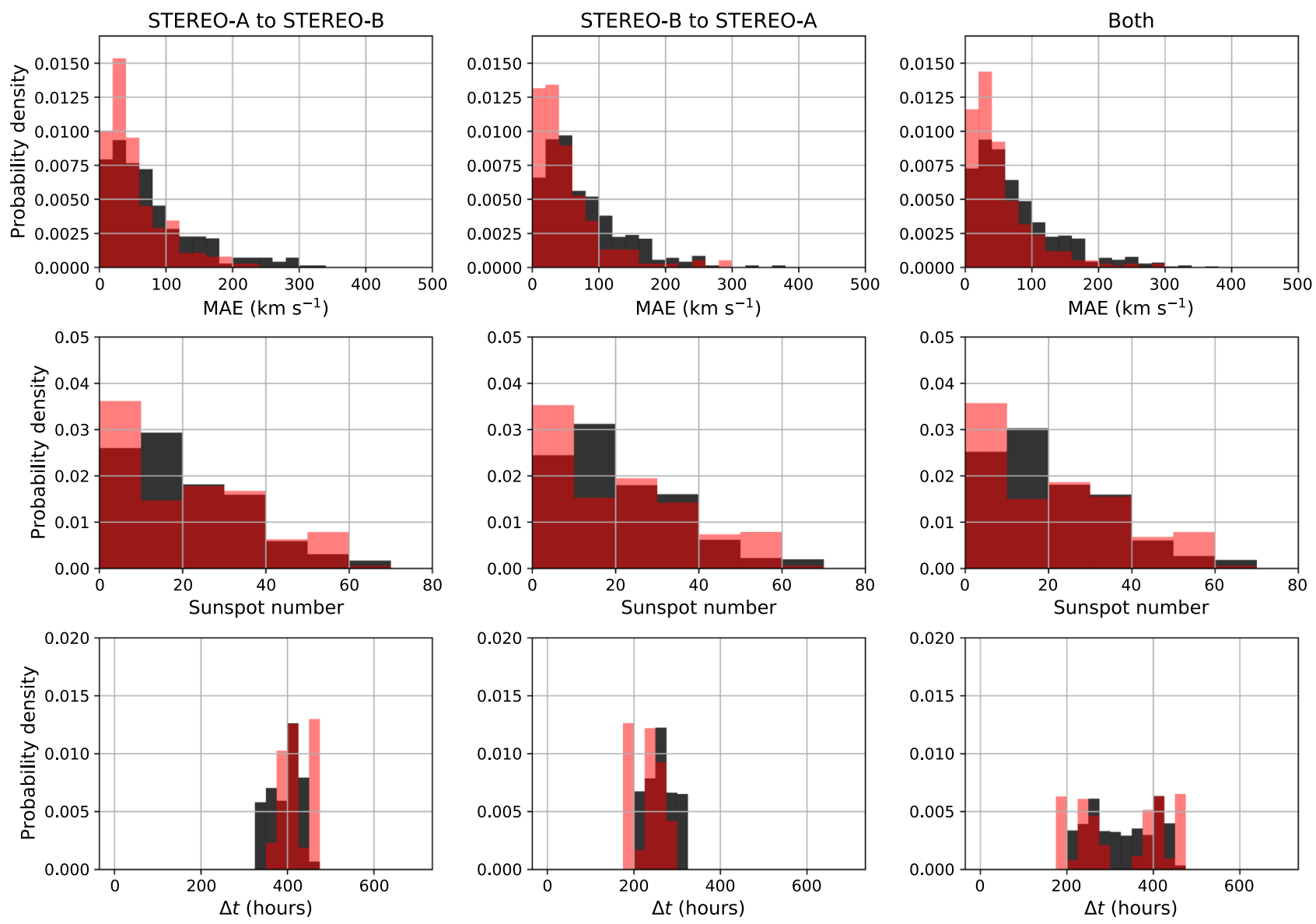

$\operatorname{High}|\Delta \theta| \quad$ Low $|\Delta \theta|$

Figure 8. Probability density for Solar Terrestrial Relations Observatory (STEREO)-A to B (left) and STEREO-B to A (middle) corotation forecasts limited to the interval August 2009 to February 2011. The right column shows the combined data set. Rows show (top) the mean absolute error (MAE) between the observed and forecast $V$, (middle) sunspot number and (bottom) $\Delta t$. The dark red areas show where there is overlap between high and low $|\Delta \theta|$.

between the two classes of $|\Delta \theta|$, as there is a marked increase in the correlation. The $p$-value represents the probability of the correlation occurring by chance, so the low $p$-value for $|\Delta \theta| \geq 6$ indicates that there is a strong and significant correlation, whereas below this, the relationship is not significant.

\section{Conclusions}

Accurate prediction of near-Earth solar wind conditions over the coming hours to days is vital for space weather forecasting and mitigation. By assuming a steady-state solar wind, longitudinally separated observations in or near the ecliptic plane can be used as a forecast for further on in the Sun's rotation. This implicitly assumes that the solar wind structures seen at both locations will be the same. The accuracy of this "corotation forecast" is affected by time evolution of the solar wind and latitudinal separation $(|\Delta \theta|)$. The error due to time evolution is itself a function of how rapidly the solar wind structure is evolving and the lead time of the forecast, which is due to the longitudinal and radial separation of the source and target spacecraft. Increased time evolution is approximately a function of solar activity, and this leads to the steady state assumption breaking down more readily, and so longer corotation times being less valid, at solar maximum. $|\Delta \theta|$ can introduce a forecast error through sampling a solar wind at the source spacecraft that is not representative of the target spacecraft. This is most important at solar minimum, where a narrow band of slow solar wind is located near the Sun's equator. 
Table 2

Averages, With Associated Standard Errors, of the Corotation Forecast $M A E$, Sunspot Number and Forecast Lead Time for the STEREO-A to B and STEREO-B to A Corotations, for Both Combined, and for all Possible Corotations Combined (i.e., Including OMNI), for the Period August 2009 to February 2011

\begin{tabular}{lcccc}
\hline & & $\begin{array}{c}\text { MAE } \\
\left(\mathrm{km} \mathrm{s}^{-1}\right)\end{array}$ & $\begin{array}{c}\text { Sunspot } \\
\text { number }\end{array}$ & $\Delta t$ (hours) \\
\hline STEREO-A to B & High $|\Delta \theta|$ & $78.8 \pm 3.5$ & $20.1 \pm 0.8$ & $395.5 \pm 1.8$ \\
& Low $|\Delta \theta|$ & $54.0 \pm 3.2$ & $19.7 \pm 1.3$ & $418.6 \pm 2.4$ \\
STEREO-B to A & High $|\Delta \theta|$ & $76.7 \pm 3.1$ & $20.3 \pm 0.8$ & $259.7 \pm 1.7$ \\
& Low $|\Delta \theta|$ & $52.6 \pm 3.7$ & $20.2 \pm 1.3$ & $235.2 \pm 2.4$ \\
Both corotations & High $|\Delta \theta|$ & $77.7 \pm 2.4$ & $20.2 \pm 0.6$ & $327.8 \pm 2.8$ \\
& Low $|\Delta \theta|$ & $53.3 \pm 2.4$ & $19.9 \pm 0.9$ & $327.1 \pm 5.0$ \\
All corotations & High $|\Delta \theta|$ & $74.0 \pm 1.6$ & $21.8 \pm 0.4$ & $325.4 \pm 3.9$ \\
& Low $|\Delta \theta|$ & $56.4 \pm 1.1$ & $18.7 \pm 0.4$ & $330.2 \pm 4.5$ \\
\hline
\end{tabular}

Abbreviations: MAE, mean absolute error; STEREO, Solar Terrestrial Relations Observatory.

Several studies have shown the effectiveness of corotation forecasts from L5 (Bailey et al., 2020; Kohutova et al., 2016; Simunac et al., 2009; Thomas et al., 2018). These studies used data from periods when combinations of the STEREO and ACE spacecraft were $60^{\circ}$ apart in heliographic longitude $(\phi)$, and showed that they outperformed the 27-day recurrence forecasts. Due to the limitation of periods where there was such a separation in $\phi$, previous analysis has mostly been restricted to solar minimum. Here, similar methods have been followed; however, the corotation forecasts have not been limited to separations of $60^{\circ}$ and so a wider range of the solar cycle has been sampled in order to fully understand the effect of $|\Delta \theta|$. Only solar wind speed has been considered here, due to its use in DA and its ability to order other solar wind parameters.

We have produced corotation forecasts using solar wind speed data from the STEREO mission and the OMNI data set. This produces six corotation configurations, mostly covering 2007 to 2014. However, a large amount of aliasing exists within this data set. As the STEREO spacecraft separate, the forecast lead time $(\Delta t)$ increases, as does the maximum $|\Delta \theta|$ and sunspot number. Therefore, it is difficult to ascribe an increase in forecast error to a single factor. At solar minimum, the solar wind is highly structured in latitude, whereas at solar maximum this structure is far more dynamic (e.g., Figure 5 of Owens, 2020). Thus, the contribution of $|\Delta \theta|$ to corotation forecast error is expected to be a strong function of the solar cycle, with largest contributions at solar minimum and smallest at solar maximum. Conversely, solar wind structure evolves much more slowly at solar minimum than solar maximum, so the contribution of $\Delta t$ to corotation forecast error is expected to be largest at solar maximum. Finally, as STEREO and OMNI spacecraft are all in the same orbital plane, the largest $|\Delta \theta|$ values are restricted to times of large longitudinal separation and hence large $\Delta t$.

The combined STEREO/OMNI data sets, however, do provide a wide range of $\Delta t$ values during both solar minimum and solar maximum even when restricted to $|\Delta \theta|<2^{\circ}$. In general, for a given $|\Delta \theta|$ value, corotation forecast MAE is higher at solar maximum than solar minimum. If the elevated MAE at solar maximum was the result of increased time-variability of ambient solar wind structures, we would expect a correlation of MAE with $\Delta t$. Instead, MAE is fairly constant across the range of $\Delta t$ available at solar maximum, suggesting the increased MAE is the result of increased frequency of transient solar wind structures at this time (Gopalswamy et al., 2009). At solar minimum, MAE increases steadily with $\Delta t$ up to around 7 days.

To attempt to isolate the $|\Delta \theta|$ contribution, we focused analysis on a period of time where sunspot number and the $\Delta t$ were fairly constant so that $|\Delta \theta|$ could be isolated. This period covered August 2009 to February 2011. Combining STEREO-A to B and B to A corotation forecasts for this period, the MAE in the corotation forecasts was significantly smaller for low $|\Delta \theta|$ periods (taken to be $<7^{\circ}$ ) than for high $|\Delta \theta|$ periods $\left(\geq 7^{\circ}\right.$ ). The mean sunspot number and $\Delta t$ values for low and high $|\Delta \theta|$ periods show no significant difference. Thus, we can attribute the difference in MAE to latitudinal offset with reasonable confidence.

Table 3

Percentage Increase (Calculated Using Equation 8) From Low to High $|\Delta \theta|$ for Average MAE, Sunspot Number and $\Delta t$ From Table 2

\begin{tabular}{lccr}
\hline & MAE $(\%)$ & Sunspot number $(\%)$ & $\Delta t(\%)$ \\
\hline STEREO-A to B & 46.0 & 2.0 & -5.5 \\
STEREO-B to A & 45.9 & 0.3 & 10.4 \\
Both corotations & 45.9 & 1.2 & 0.2 \\
All corotations & 31.4 & 16.7 & -1.5 \\
\hline
\end{tabular}

Note. This covers the period August 2009 to February 2011. Abbreviations: MAE, mean absolute error; STEREO, Solar Terrestrial Relations Observatory.
Looking in more detail at this $8 / 2009$ to $2 / 2011$ period, there is a strong correlation between $|\Delta \theta|$ and MAE for $|\Delta \theta|>6^{\circ}$, but not for $|\Delta \theta| \geq 6^{\circ}$. At around this same latitudinal separation value, the observed forecast MAE becomes comparable to that expected from heliospheric modeling, where only the latitudinal effect is present (i.e., there is no time evolution and no solar wind transients Owens et al., 2020). See the gray curve in Figure 6. Thus, we suggest that for $|\Delta \theta|<6^{\circ}$, the latitudinal offset error in corotation forecasts is present, but is not detectable due to other factors (such as time evolution and CMEs) dominating. For $|\Delta \theta| \geq 6^{\circ}$, however, the latitudinal offset is the primary source of corotation forecast error and the magnitude is in good agreement with that expected from steadystate solar wind modeling. These findings are broadly consistent with studies qualitatively looking at SIRs (e.g., Jian et al., 2019). This explains 
Table 4

Linear Correlation Coefficients and Corresponding p-Values for MAE and $|\Delta \theta|$, Split Into $|\Delta \theta|<6$ and $|\Delta \theta| \geq 6$

\begin{tabular}{lcc}
\hline & Correlation coefficient & $p$-value \\
\hline$|\Delta \theta|<6$ & 0.40 & 0.44 \\
$|\Delta \theta| \geq 6$ & 0.75 & 0.02 \\
\hline
\end{tabular}

Note. This uses all corotations and covers the period from August 2009 to February 2011. The $p$-value represents the probability of the correlation occurring by chance.

Abbreviations: MAE, mean absolute error; STEREO, Solar Terrestrial Relations Observatory. why a latitudinal effect is not detectable in analysis of 27-day recurrence forecasts. The maximum change in latitude for a single spacecraft in the ecliptic plane from one Carrington rotation to the next is $3.5^{\circ}$, hence the signal not being present. This also implies that for using observations in DA, if $|\Delta \theta|<6$, observation errors could be assumed constant, where above this, the observational error would be dependent on $|\Delta \theta|$.

Typically, observations with $|\Delta \theta|<6$ would be preferable for DA and corotation forecasting, as at this separation, the latitudinal effect is minimized in comparison with other sources of error. This finding has implications on the use of future L5 mission data. L5 reaches a maximum $|\Delta \theta|$ of around $5^{\circ}$ with Earth (at times close to the summer and winter solstices), meaning that the latitudinal variation can be largely disregarded. Owens et al. (2019) showed that there is a time-of-year variation in the modeled impact of $|\Delta \theta|$ on MAE. This is not investigated here due to data limitations.

The future space weather monitoring mission to the L5 Lagrange point offers a new opportunity for corotation forecasts for the solar wind. The investigation into the effect of $|\Delta \theta|$ on forecast error here has found that for $|\Delta \theta|<6$, there is a minimal impact due to other sources of error. However, for $|\Delta \theta| \geq 6$, the error contribution increases and there is a clear relationship between $|\Delta \theta|$ and forecast MAE. Due to the maximum $|\Delta \theta|$ between L5 and Earth being $5^{\circ}$, this result implies that the effect from $|\Delta \theta|$ on the forecast error would be minimal.

Moving forward, this work can aid the effective use of observations in DA for forecasting the solar wind. It will enable more accurate observation error covariances to be calculated when there is a $|\Delta \theta|$ between observations and Earth. Furthermore, this will allow observational errors that result from $|\Delta \theta|$ to be corrected, ensuring the DA methodologies perform optimally.

\section{Data Availability Statement}

These data were downloaded from the OMNIWeb portal at https://omniweb.gsfc.nasa.gov/ow.html, and accessed through the HelioPy module in Python https://docs.heliopy.org/en/0.6.7/. STEREO-A and STEREO-B data were downloaded from the CDAWeb Data Explorer portal at https://cdaweb.gsfc.nasa.gov/ cgi-bin/eval2.cgi?dataset=STA_COHO1HR_MERGED_MAG_PLASMA\&index=sp_phys, but accessed through HelioPy. Spacecraft location data were downloaded from https://omniweb.gsfc.nasa.gov/coho/helios/heli.html. HelioMAS model output is available from the Predictive Science Inc. (website: http://www. predsci.com/mhdweb/home.php).

\section{Acknowledgments}

The authors are grateful to the Space Physics Data Facility and National Space Science Data Center for OMNI. $\mathrm{H}$. Turner is funded through SCENARIO grant number NE/S007261/1. Work was part-funded by Science and Technology Facilities Council (STFC) grant numbers ST/R000921/1 and ST/ V000497/1, and Natural Environment Research Council (NERC) grant number NE/S010033/1.

\section{References}

Akioka, M., Nagatsuma, T., Miyake, W., Ohtaka, K., \& Marubashi, K. (2005). The L5 mission for space weather forecasting. Advances in Space Research, 35(1), 65-69. https://doi.org/10.1016/j.asr.2004.09.014

Allen, R. C., Ho, G. C., Jian, L. K., Mason, G. M., Vines, S. K., \& Lario, D. (2020). Predictive capabilities and limitations of stream interaction region observations at different solar longitudes. Space Weather, 18(4). https://doi.org/10.1029/2019SW002437

Allen, R. C., Ho, G. C., Mason, G. M., Li, G., Jian, L. K., Vines, S. K., et al. (2021). Radial evolution of a CIR: Observations from a nearly radially aligned event between Parker Solar Probe and STEREO-A. Geophysical Research Letters, 48(3). https://doi.org/10.1029/2020GL091376

Alves, M. V., Echer, E., \& Gonzalez, W. D. (2006). Geoeffectiveness of corotating interaction regions as measured by Dst index. Journal of Geophysical Research, 111(7), 1-9. https://doi.org/10.1029/2005JA011379

Bailey, R. L., Möstl, C., Reiss, M. A., Weiss, A. J., Amerstorfer, U. V., Amerstorfer, T., et al. (2020). Prediction of Dst during solar minimum using in situ measurements at L5. Space Weather, 18(5), 1-12. https://doi.org/10.1029/2019SW002424

Cannon, P. S. (2013). Extreme space weather-A report published by the UK royal academy of engineering. Space Weather, 11(4), 138-139. https://doi.org/10.1002/swe.20032

Case, A. W., Spence, H. E., Owens, M. J., Riley, P., \& Odstrcil, D. (2008). Ambient solar winds' effect on ICME transit times. Geophysical Research Letters, 35(15), 1-5. https://doi.org/10.1029/2008GL034493

Davies, J. (2020). The COR and HI instruments for the Lagrange L5 mission. In The COR and HI instruments for the Lagrange L5 mission.

Galvin, A. B., Kistler, L. M., Popecki, M. A., Farrugia, C. J., Simunac, K. D., Ellis, L., et al. (2008). The plasma and suprathermal ion composition (PLASTIC) investigation on the STEREO observatories. Space Science Reviews, 136(1-4), 437-486. https://doi.org/10.1007/s11214-007-9296-X 
Gopalswamy, N., Yashiro, S., Michalek, G., Stenborg, G., Vourlidas, A., Freeland, S., \& Howard, R. (2009). The SOHO/LASCO CME catalog. Earth, Moon, and Planets, 104(1-4), 295-313. https://doi.org/10.1007/s11038-008-9282-7

Gosling, J. T. (1993). The solar flare myth. Journal of Geophysical Research, 98(A11), 18937-18949. https://doi.org/10.1029/93ja01896

Janjić, T., Bormann, N., Bocquet, M., Carton, J. A., Cohn, S. E., Dance, S. L., et al. (2018). On the representation error in data assimilation. Quarterly Journal of the Royal Meteorological Society, 144(713), 1257-1278. https://doi.org/10.1002/qj.3130

Jian, L. K., Luhmann, J. G., Russell, C. T., \& Galvin, A. B. (2019). Solar Terrestrial Relations Observatory (STEREO) observations of stream interaction regions in 2007-2016: Relationship with heliospheric current sheets, solar cycle variations, and dual observations. Solar Physics, 294(3). https://doi.org/10.1007/s11207-019-1416-8

Kaiser, M. L., Kucera, T. A., Davila, J. M., Cyr, O. C., Guhathakurta, M., \& Christian, E. (2008). The STEREO mission: An introduction. Space Science Reviews, 136(1-4), 5-16. https://doi.org/10.1007/s11214-007-9277-0

Kohutova, P., Bocquet, F. X., Henley, E. M., \& Owens, M. J. (2016). Improving solar wind persistence forecasts: Removing transient space weather events, and using observations away from the Sun-Earth line. Space Weather, 14(10), 802-818. https://doi. org/10.1002/2016SW001447

Lang, M., Browne, P., van Leeuwen, P. J., \& Owens, M. (2017). Data assimilation in the solar wind: Challenges and first results. Space Weather, 15(11), 1490-1510. https://doi.org/10.1002/2017SW001681

Lang, M., \& Owens, M. J. (2019). A variational approach to data assimilation in the solar wind. Space Weather, 17(1), 59-83. https://doi. org/10.1029/2018SW001857

Lang, M., Witherington, J., Owens, M. J., \& Turner, H. (2021). Improving solar wind forecasting using data assimilation. Space Weather, 1-23. https://doi.org/10.1029/2020sw002698

Linker, J. A., Mikić, Z., Biesecker, D. A., Forsyth, R. J., Gibson, S. E., Lazarus, A. J., et al. (1999). Magnetohydrodynamic modeling of the solar corona during Whole Sun Month. Journal of Geophysical Research, 104(A5), 9809-9830. https://doi.org/10.1029/1998JA900159. Retrieved from https://onlinelibrary.wiley.com/doi/10.1029/1998JA900159

McComas, D. J., Elliott, H. A., Schwadron, N. A., Gosling, J. T., Skoug, R. M., \& Goldstein, B. E. (2003). The three-dimensional solar wind around solar maximum. Geophysical Research Letters, 30(10), 1. https://doi.org/10.1029/2003gl017136

Owens, M. J. (2018). Time-window approaches to space-weather forecast metrics: A solar wind case study. Space Weather, 16(11), 18471861. https://doi.org/10.1029/2018SW002059

Owens, M. J. (2020). Solar-wind structure (No. July). In Oxford Research Encyclopedia of Physics. https://doi.org/10.1093/ acrefore/9780190871994.013.19

Owens, M. J., Challen, R., Methven, J., Henley, E., \& Jackson, D. R. (2013). A 27 day persistence model of near-Earth solar wind conditions: A long lead-time forecast and a benchmark for dynamical models. Space Weather, 11(5), 225-236. https://doi.org/10.1002/swe.20040

Owens, M. J., Lang, M., Riley, P., Lockwood, M., \& Lawless, A. S. (2020). Quantifying the latitudinal representivity of in situ solar wind observations. Journal of Space Weather and Space Climate, 10, 8. https://doi.org/10.1051/swsc/2020009

Owens, M. J., Riley, P., Lang, M., \& Lockwood, M. (2019). Near-earth solar wind forecasting using corotation from L5: The error introduced by heliographic latitude offset. Space Weather, 17(7), 1105-1113. https://doi.org/10.1029/2019SW002204

Parker, E. N. (1958). Dynamics of the interplanetary gas and magnetic fields. The Astrophysical Journal, 128, 664-676. https://doi. org/10.1086/146579. Retrieved from https://www.sci-hub.tw/10.1002/iroh.19620470121

Perrone, D., Stansby, D., Horbury, T. S., \& Matteini, L. (2019). Radial evolution of the solar wind in pure high-speed streams: HELIOS revised observations. Monthly Notices of the Royal Astronomical Society, 483(3), 3730-3737. https://doi.org/10.1093/mnras/sty3348

Reiss, M. A., Temmer, M., Veronig, A. M., Nikolic, L., Vennerstrom, S., Schöngassner, F., \& Hofmeister, S. J. (2016). Verification of high-speed solar wind stream forecasts using operational solar wind models. Space Weather, 14(7), 495-510. https://doi.org/10.1002/2016SW001390

Richardson, I. G., \& Cane, H. V. (2012). Solar wind drivers of geomagnetic storms during more than four solar cycles. Journal of Space Weather and Space Climate, 2, 1-9. https://doi.org/10.1051/swsc/2012001

Riley, P., Linker, J. A., Lionello, R., \& Mikic, Z. (2012). Corotating interaction regions during the recent solar minimum: The power and limitations of global MHD modeling. Journal of Atmospheric and Solar-Terrestrial Physics, 83, 1-10. https://doi.org/10.1016/j. jastp.2011.12.013

Simunac, K. D., Kistler, L. M., Galvin, A. B., Popecki, M. A., \& Farrugia, C. J. (2009). In situ observations from STEREO/PLASTIC: A test for L5 space weather monitors. Annales Geophysicae, 27(10), 3805-3809. https://doi.org/10.5194/angeo-27-3805-2009

Stone, E. C., Frandsen, A. M., Mewaldt, R. A., Christian, E. R., Margolies, D., Ormes, J. F., \& Snow, F. (1998). The advanced composition explorer. Space Science Reviews, 86, 1-22. https://doi.org/10.1023/A:1005082526237

Thomas, S. R., Fazakerley, A., Wicks, R. T., \& Green, L. (2018). Evaluating the skill of forecasts of the near-earth solar wind using a space weather monitor at L5. Space Weather, 16(7), 814-828. https://doi.org/10.1029/2018SW001821

Vokhmyanin, M. V., Stepanov, N. A., \& Sergeev, V. A. (2019). On the evaluation of data quality in the OMNI interplanetary magnetic field database. Space Weather, 17(3), 476-486. https://doi.org/10.1029/2018SW002113

Wang, Y.-M., \& Sheeley, N. R., Jr (1991). Magnetic flux transport and the sun's dipole moment-New twists to the Babcock-Leighton model. The Astrophysical Journal, 375(1), 761. https://doi.org/10.1086/170240. Retrieved from http://thesis.ekt.gr/thesisBookReader/ id/1834\{\\#\}page/104/mode/2uphttp://adsabs.harvard.edu/doi/10.1086/170240

Wijsen, N., Samara, E., Aran, À., Lario, D., Pomoell, J., \& Poedts, S. (2021). A self-consistent simulation of proton acceleration and transport near a high-speed solar wind stream. The Astrophysical Journal, 908(2), L26. https://doi.org/10.3847/2041-8213/abe1cb 